\title{
Pulsed Electromagnetic Field Stimulation Promotes Anti-cell Proliferative Activity in Doxorubicin-treated Mouse Osteosarcoma Cells
}

\author{
YOSHITAKA MURAMATSU ${ }^{1}$, TAKUYA MATSUI ${ }^{2}$, MASATAKA DEIE $^{1}$ and KEIJI SATO ${ }^{1}$ \\ ${ }^{I}$ Department of Orthopaedic Surgery, Aichi Medical University School of Medicine Nagakute, Aichi, Japan \\ ${ }^{2}$ Department of Physiology, Aichi Medical University School of Medicine Nagakute, Aichi, Japan
}

\begin{abstract}
Aim: We aimed to investigate the synergistic effects of pulsed electromagnetic field (PEMF) and doxorubicin therapy in a mouse osteosarcoma cell line (LM8 cells) in vitro. Materials and Methods: The effects of PEMF $(5 \mathrm{mT}, 200 \mathrm{~Hz})$ of different durations and doxorubicin on the proliferative activity of LM8 cells were measured by the MTT assay. Apoptotic-related factors such as cell-cycle phase, mitochondrial membrane potential, and caspase 3/7 activity were investigated using 4',6-diamidino-2-phenylindole staining and apoptosis kits. Identification of intracellular signaling molecules induced by the combination was comprehensively explored using a stress and apoptosis-related protein array kit. Results: PEMF enhanced the inhibition of cell proliferation mediated by doxorubicin but did not affect the cell cycle, mitochondrial membrane potential, or doxorubicin-induced $G_{2} / M$ arrest. The combination of PEMF and doxorubicin altered a few signaling molecules. PEMF tended to reduce the doxorubicin-induced decrease of phosphorylated $B A D$, while reducing the increased expression of total $I K B$ and phosphorylated-CHKI induced by doxorubicin. Conclusion: Our results indicate that combination of PEMF and doxorubicin could be a novel chemotherapeutic strategy.
\end{abstract}

Previous reports have investigated the promotion of bone formation via electrical stimulation with direct current, alternating current, and electromagnetic field stimulation (14). Jansen $\mathrm{JH}$ et al. revealed that pulsed electromagnetic field (PEMF)-induced human osteogenic differentiation was

This article is freely accessible online.

Correspondence to: Professor Masataka Deie, Department of Orthopaedic Surgery, Aichi Medical University School of Medicine, Nagakute, Aichi 480-1195, Japan. E-mail: snm3@aichi-med-u.ac.jp

Key Words: PEMF, doxorubicin, osteosarcoma, cell proliferation, synergistic effect. unrelated to the phosphorylation of ERK1/2 in an in vitro study (5). Although the mechanism of bone healing by PEMF is not clear, PEMF instruments are commercially available for bone fracture treatment. Additionally, it is predicted that PEMF could be applicable to cancer therapy. An increasing number of reports indicate that PEMF affects intracellular signaling molecules and invasion factors in various cancer types, such as chondrosarcoma, neuroblastoma, and breast cancer cells (6-8). Osteosarcoma is the most common primary malignant bone tumor. The current standard for neoadjuvant chemotherapy for patients with osteosarcoma is doxorubicinbased combination chemotherapy. However, to our knowledge, there are no reports on PEMF and doxorubicin combination therapy for osteosarcoma.

We previously showed that PEMF stimulation regulates cell growth and doxorubicin-induced cytotoxicity effects in multidrug-resistant tumor cells that express P-glycoproteins (9). Furthermore, we have been investigating the mechanisms of anticancer effects induced by PEMF. We obtained evidence that PEMF does not affect adhesion molecules, such as integrin, and regulates the expression of some transient receptor potential genes $(10,11)$. However, the effects of PEMF alone against osteosarcoma are not fully understood.

In this study, we aimed to investigate the synergistic effect of PEMF and doxorubicin and seek apoptotic-related factors.

\section{Materials and Methods}

Cell lines and culture. Mouse osteosarcoma cell line LM8 was obtained from the Japanese Collection of Research Bioresources cell bank (Oasaka, Japan), and maintained in Eagle's minimal essential medium (Sigma-Aldrich, St Louis, MO, USA) containing 10\% (v/v) heat-inactivated fetal calf serum (Gibco Thermo Fisher Scientific, Waltham, MA, USA), $100 \mathrm{U} / \mathrm{ml}$ penicillin (Gibco), and $100 \mathrm{mg} / \mathrm{ml}$ streptomycin (Gibco) at $37^{\circ} \mathrm{C}$ in a humidified incubator with $5 \% \mathrm{CO}_{2}$.

PEMF-generating system. The PEMF was generated using a PEMF stimulator system (RIKEN Model 021A; Satelit, Tokyo, Japan) (Figure 1). The experimental conditions were according to a report by Zhang et al. (10). 
Cell proliferation assays. LM8 cells were seeded onto 96-well plates at a density of $2 \times 10^{4}$ cells per well. After overnight incubation, cells were treated with PEMF $(5 \mathrm{mT}, 200 \mathrm{~Hz})$ for 1 and $12 \mathrm{~h}$ or following $24 \mathrm{~h}$ of culture with/without doxorubicin (Kyowa Hakko Kirin, Tokyo, Japan) at defined concentrations $(0.05,0.1,0.25,0.5,1,5,50 \mathrm{ug} / \mathrm{ml})$. WST-8 Reagent (Cell Counting kit; Dojindo Lab. Tokyo, Japan) was added to each well and cells were further cultured for $2 \mathrm{~h}$ according to the manufacturer's instructions. Absorbance in conditioned medium was monitored at $490 \mathrm{~nm}$ using a microplate reader (Molecular Devices, Sunnyvale, CA, USA). Half maximal (50\%) of inhibitory concentrations $\left(\mathrm{IC}_{50}\right)$ values were calculated using Softmax Pro6 (Molecular Devices). The untreated cells as control were defined as $100 \%$. These measurements were performed in five independent.

Uptake of doxorubicin. LM8 cells were seeded in 6-well plates at $3 \times 10^{5}$ cells. After overnight incubation, doxorubicin (Kyowa Hakko Kirin) at $0.5 \mathrm{ug} / \mathrm{ml}$, which was defined using the results of $\mathrm{IC}_{50}$, was added to each well. Treated cells were stimulated with/wihout PEMF for 1, and $12 \mathrm{~h}$ following $24 \mathrm{~h}$ of culture. LM8 cells in 6-well plates were collected with trypsin, and doxorubicin uptake was assayed by flow cytometry. In briefly, the collected cells were fixed with $10 \%$ formalin buffer. And the intranuclear fluorescence intensity of doxorubicin was measured by a FACS Canto II with 488-nm excitation. The untreated cells as reference control were defined as baseline levels. These measurements were performed in five independent

Analysis of cell cycle. Cells were seeded at $3 \times 10^{5}$ cells/well in 6-well plates. After culturing the cells after 1 and $12 \mathrm{~h}$ of PEMF, cells were trypsinized from the plate, fixed in $70 \%$ ethanol, and treated with $0.5 \%$ ribonuclease for $30 \mathrm{~min}$. DNA was stained with 4',6diamidiono-2-phenylindole (DAPI, $1.2 \mu \mathrm{M}$ ) for $30 \mathrm{~min}$ at room temperature in the dark. Flow cytometry and cell cycle analysis were performed using a FACS Canto II with 405-nm excitation. At least 10,000 gated cells were examined per cycle. The untreated cells were defined as reference control. These measurements were performed in five independent.

Measurement of mitochondrial membrane potential. LM8 cells were seeded onto 96-well plates at a density of $4 \times 10^{4}$ cells per well. After overnight incubation, doxorubicin $(0.5 \mu \mathrm{g} / \mathrm{ml})$ was added to the cells and they were immediately treated with PEMF for 1 and $12 \mathrm{~h}$. The kinetics of the mitochondrial membrane potential in the cells were measured using a membrane potential cytotoxicity kit (Mito-ID; Enzo Life Science International, PA, USA) and observed by fluorescence microscopy (IX73; Olympus, Tokyo, Japan). The untreated cells were defined as reference control. These measurements were performed in five independent.

Measurement of caspase 3/7 activities. LM8 cells were seeded onto 96-well plates at a density of $4 \times 10^{4}$ cells per well. After overnight incubation, doxorubicin $(0.5 \mu \mathrm{g} / \mathrm{ml})$ that was defined using the results of $\mathrm{IC}_{50}$, and PEMF were applied to the cells. Cell lysates for caspase $3 / 7$ activities were obtained 12 and $24 \mathrm{~h}$ after treatment with doxorubicin with/wtihout PEMF. Caspase 3/7 activity was determined using Caspase-Glo 3/7 Assay kit (Promega Co., Madison, WI, USA), according to the manufacturer's protocol. The untreated cells were defined as reference control. These measurements were performed in five independent.

Measurement of intracellular $\mathrm{Ca}^{2+}$ concentrations. LM8 cells were seeded onto 96-well plates at a density of $4 \times 10^{4}$ cells per well. After overnight incubation, doxorubicin $(0.5 \mu \mathrm{g} / \mathrm{ml})$ was applied to cells and they were immediately treated with PEMF for 1 and $12 \mathrm{~h}$. After treatment, cells were incubated with Fluo-4 solution (Fluo-4 MW calcium assay kit; Invitrogen, Thermo Fisher Scientific, Carlsbad, CA, USA) in Hanks' balanced salt solution buffer for $30 \mathrm{~min}$ at $37^{\circ} \mathrm{C}$. The cytosol $\mathrm{Ca}^{2+}$ concentrations were measured using a microplate reader (Molecular Devices). The untreated cells were defined as reference control. These measurements were performed in five independent.

Screening intracellular signaling molecules. LM8 cells were seeded onto 96-well plates at a density of $4 \times 10^{4}$ cells per well. After overnight incubation, doxorubicin $(0.5 \mu \mathrm{g} / \mathrm{ml})$ was applied to the cells and they were immediately treated with PEMF for $12 \mathrm{~h}$. Trypsinized cells were rinsed with cold phosphate-buffered saline and immediately solubilized in cell lysate buffer (Cell Signaling Technology, Danvers, MA, USA) containing complete protease inhibitor cocktail (Roche Diagnostics Ltd., Sandhofer Str. Germany) and phenylmethylsulfonyl fluoride at $1 \mathrm{mM}$ (Sigma-Aldrich) by blocking the lysates gently at $4^{\circ} \mathrm{C}$ for $30 \mathrm{~min}$. Following microcentrifugation at $14,000 \times g$ for 5 min, supernatants were transferred into a clean test tube and sample proteins $(1.0 \mathrm{mg} / \mathrm{ml})$ were diluted and incubated with the PathScan Stress and Apoptosis Signaling Antibody Array Kit (Cell Signaling Technology) as per the manufacturer's instructions. The expressed dots were visualized using LumiGlo reagent and detected by the ImageQuant LAS-4000 (GE Healthcare Co. Wauwatosa, WI, USA). Dot densities were measured using Image $J$ (National Institutes of Health, Bethesda, MD, USA) and corrected to the relative density of $\alpha$-tubulin. The untreated cells were defined as reference control. These measurements were performed in four independent.

Statistical analysis. Statistical analysis was performed by the MannWhitney $U$-test to compare the data against negative controls using SPSS version 22 (IBM., Armonk, NY, USA). Results are expressed as means \pm S.D. $p$-Values less than 0.05 were considered statistically significant.

\section{Results}

Antiproliferative effects of the combination of PEMF and doxorubicin. The possibility of PEMF affecting the intracellular microenvironment of LM8 cells has been previously raised $(10,12)$; thus, we evaluated whether PEMF would accelerate the pharmacological activity of doxorubicin. In this study, the doxorubicin-treated cells immediately after PEMF stimulation for $1 \mathrm{~h}$ did not change regarding cell proliferation. Thus, this $\mathrm{IC}_{50}$ was not clear. While, the proliferation of cells treated for $12 \mathrm{~h}$ was dose-dependently reduced (Table I). But antiproliferative effects of the cells followed for $24 \mathrm{~h}$ after PEMF stimulation were not found changed between PEMF treatment and non-treatment. PEMF stimulation significantly enhanced this effect (Table I).

To verify the doxorubicin-enhancing effects of PEMF, doxorubicin uptake in LM8 cells was measured by FACS. PEMF stimulation of LM8 cells weakly promoted doxorubicin uptake (Figure 2A).

The results above indicated that PEMF promoted the uptake of doxorubicin, hence we investigated the effects of 

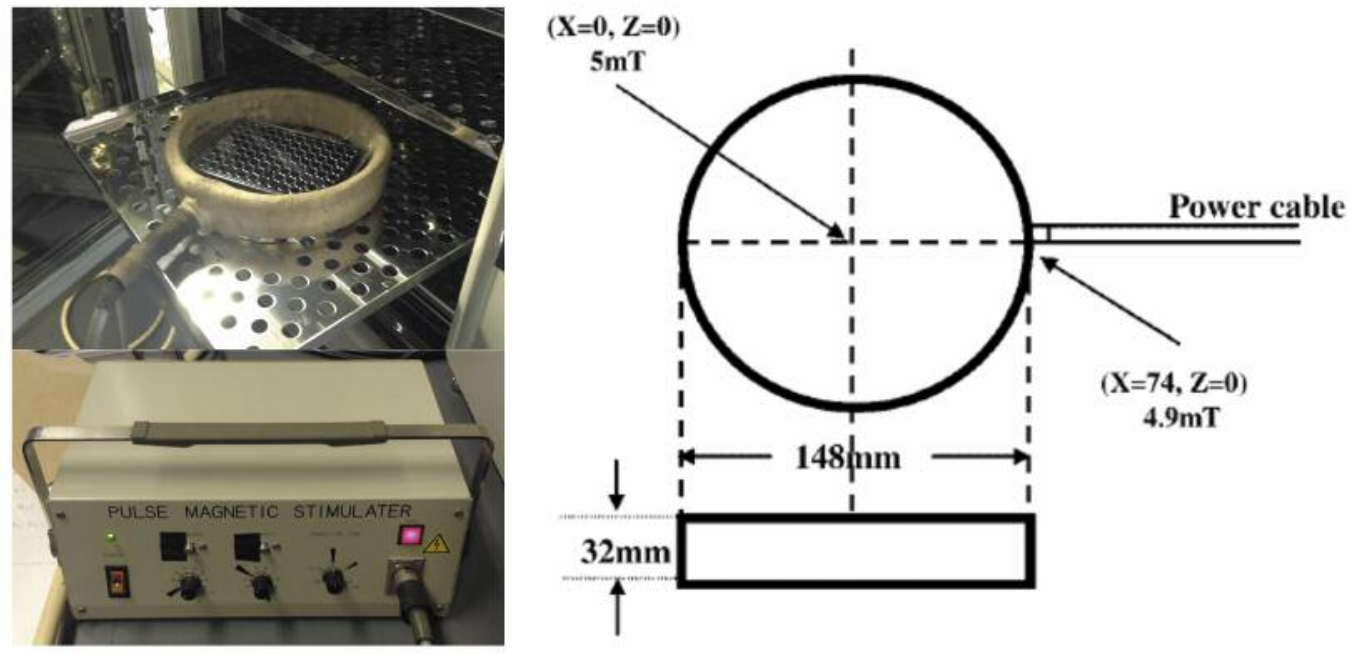

Figure 1. Generating apparatus.

doxorubicin with/without PEMF on the cell cycle. Stimulation with PEMF alone for $12 \mathrm{~h}$ reduced the population of cells at $\mathrm{S}$-phase, while doxorubicin induced $\mathrm{G}_{2} / \mathrm{M}$ arrest at $12 \mathrm{~h}$, but not at $1 \mathrm{~h}$ (Figure 2B). However, cell treatment with a combination of PEMF and doxorubicin did not alter the cellcycle distribution, similarly to cells treated with doxorubicin alone.

We investigated whether the combination of PEMF and doxorubicin induced apoptosis. First, we measured the mitochondrial membrane potential, which are involved in regulation of $\left[\mathrm{Ca}^{2+}\right] \mathrm{i}$ and apoptosis. Neither PEMF nor doxorubicin significantly altered the mitochondrial membrane potential at $1 \mathrm{~h}$ or $12 \mathrm{~h}$ (Figure 2C).

Intracellular apoptosis molecules. We investigated whether the antiproliferative activity of PEMF and doxorubicin combined was linked to intracellular molecules. We focused on a key player $\mathrm{Ca}^{2+}$. The level of intracellular $\mathrm{Ca}^{2+},\left[\mathrm{Ca}^{2+}\right] \mathrm{i}$ at 1 and $12 \mathrm{~h}$ after PEMF treatment, alone and in combination with doxorubicin, was significantly higher in comparison with non-treated cells. However, the $\left[\mathrm{Ca}^{2+}\right] \mathrm{i}$ level of cells treated with doxorubicin alone was practically the same as that of non-treated cells. Thus, changes in $\left[\mathrm{Ca}^{2+}\right] \mathrm{i}$ were caused by PEMF but not by doxorubicin (Figure 3A). Furthermore, we determined the activity of caspase $3 / 7$, which is an apoptotic indicator. Treatment of LM8 cells with doxorubicin alone for 24 h significantly enhanced caspase $3 / 7$ activity, while PEMF alone had no effect. Interestingly, PEMF stimulation significantly reduced the enhancement of caspase $3 / 7$ activity by doxorubicin at $24 \mathrm{~h}$ (Figure 3B).

Comprehensive analysis of intracellular signaling molecules. We explored other intracellular signaling molecules in addition
Table I. Half-maximal (50\%) of inhibitory concentrations $\left(I_{50}\right)$ for doxorubicin (DOX) for combination of pulsed electromagnetic field (PEMF) and doxorubicin (DOX) treatment of LM8 cells. The IC 50 was investigated immediately and at $24 \mathrm{~h}$ after pulsed electromagnetic field (PEMF) stimulation for 1 and $12 \mathrm{~h}$. The data represent the means $\pm S . D$. of four independent experiments. ${ }^{*} p<0.05 \mathrm{vs}$. Dox-treated non-PEMFtreated cells at each times.

\begin{tabular}{lcccc}
\hline & \multicolumn{4}{c}{$\mathrm{IC}_{50}$ DOX $(\mu \mathrm{g} / \mathrm{ml})$} \\
\cline { 2 - 5 } & \multicolumn{2}{c}{$\begin{array}{c}\text { Immediately after PEMF } \\
\text { stimualtion }\end{array}$} & \multicolumn{2}{c}{$\begin{array}{c}\text { 24 h after PEMF } \\
\text { stimulation }\end{array}$} \\
\cline { 2 - 5 } Treatment & $1 \mathrm{~h}$ & $12 \mathrm{~h}$ & $1 \mathrm{~h}$ & $12 \mathrm{~h}$ \\
\hline DOX alone & $>5$ & $1.510 \pm 0.250$ & $0.773 \pm 0.049$ & $0.426 \pm 0.069$ \\
DOX+PEMF & $>5$ & $0.655 \pm 0.095^{*}$ & $0.753 \pm 0.082$ & $0.419 \pm 0.045$ \\
\hline
\end{tabular}

to $\mathrm{Ca}^{2+}$ and caspase $3 / 7$ using protein arrays (Figure $4 \mathrm{~A}$ ). Doxorubicin treatment alone significantly enhanced the levels of total IKB and phosphorylated CHK1, and significantly reduced the levels of phosphorylated AKT and BAD in LM8 cells. Moreover, PEMF alone tended to reduce the levels of phosphorylated-AKT, -BAD and - transforming growth factor $\beta$-activated kinase 1 (TAK1) that is defined as mitogenactivated protein kinase kinase kinase that can be activated by TGF- $\beta$. PEMF and doxorubicin co-treatment further tended to reduce the levels of phosphorylated BAD compared with doxorubicin or PEMF alone. In addition, PEMF decreased the up-regulated levels of total IkB and phosphorylated CHK1 mediated by doxorubicin (Figure 4B). 
A

$1 \mathrm{H}$

$12 \mathrm{H}$
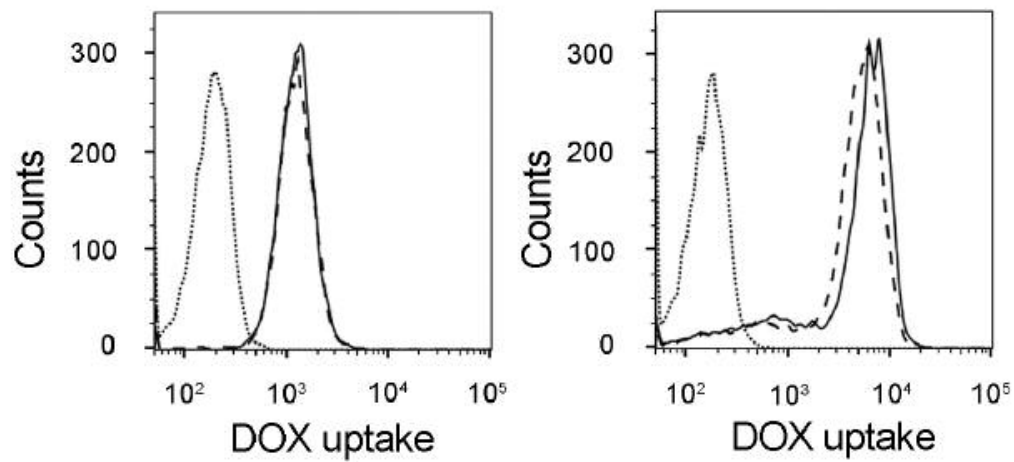

DOX

PEMF

B

$1 \mathrm{H}$

$12 \mathrm{H}$

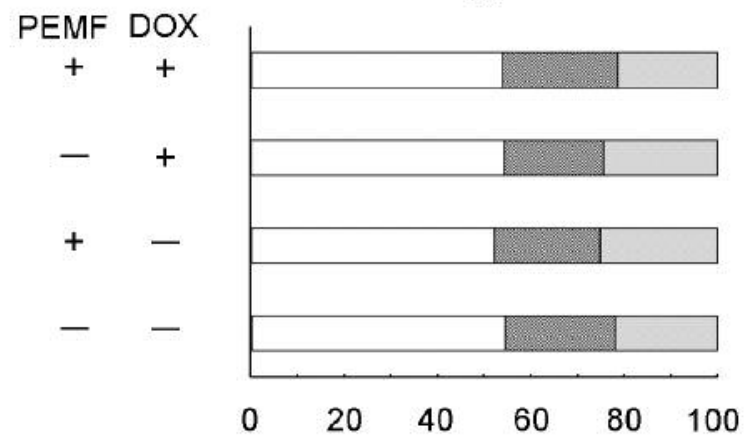

$\%$ Cell population

$\%$ Cell population

C
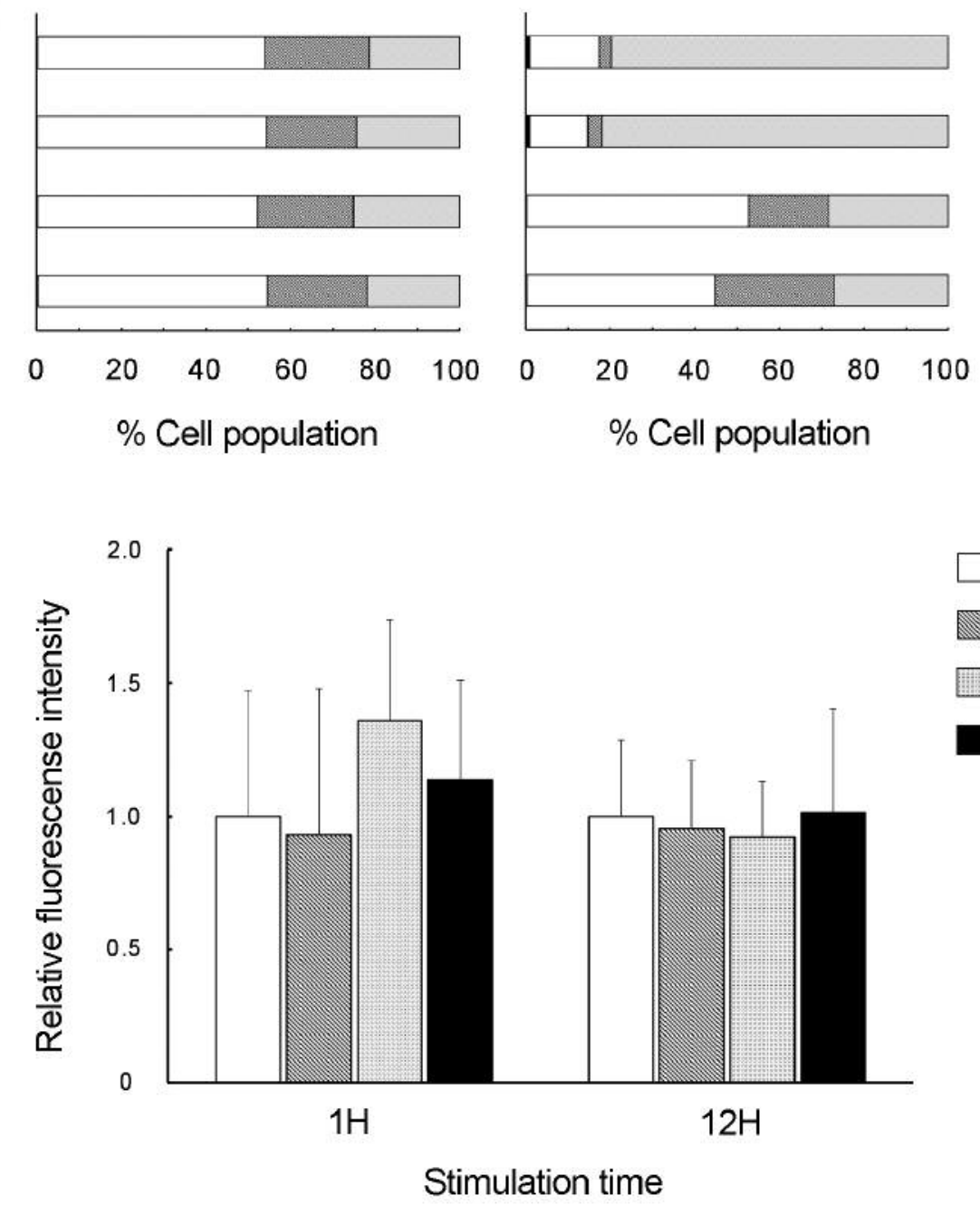

- SubG $_{1}$ $\mathrm{G}_{0} / \mathrm{G}_{1}$ $S$ $\mathrm{G}_{2} / \mathrm{M}$

$\square$ Control Inis PEMF alone $\square$ DOX alone - PEMF and DOX

Stimulation time

Figure 2. Biological effects of pulsed electromagnetic field (PEMF) and doxorubicin (DOX) on LM8 cells. A: PEMF tended to promote DOX uptake for 12 h. B: The cell populations at different cell-cycle phases after treatment of LM8 cells with the combination of PEMF and DOX, PEMF alone, and DOX alone in LM8 cells. Cells were stained with 4',6-diamidiono-2-phenylindole solution after fixation by 70\% ethanol, and analyzed by flow cytometry. $C$ : The fluorescence intensities reflecting mitochondrial membrane potential were evaluated after treatment of LM8 cells with a combination of PEMF and DOX, PEMF alone, and DOX alone. The data represent the means \pm S.D. of five independent experiments. 

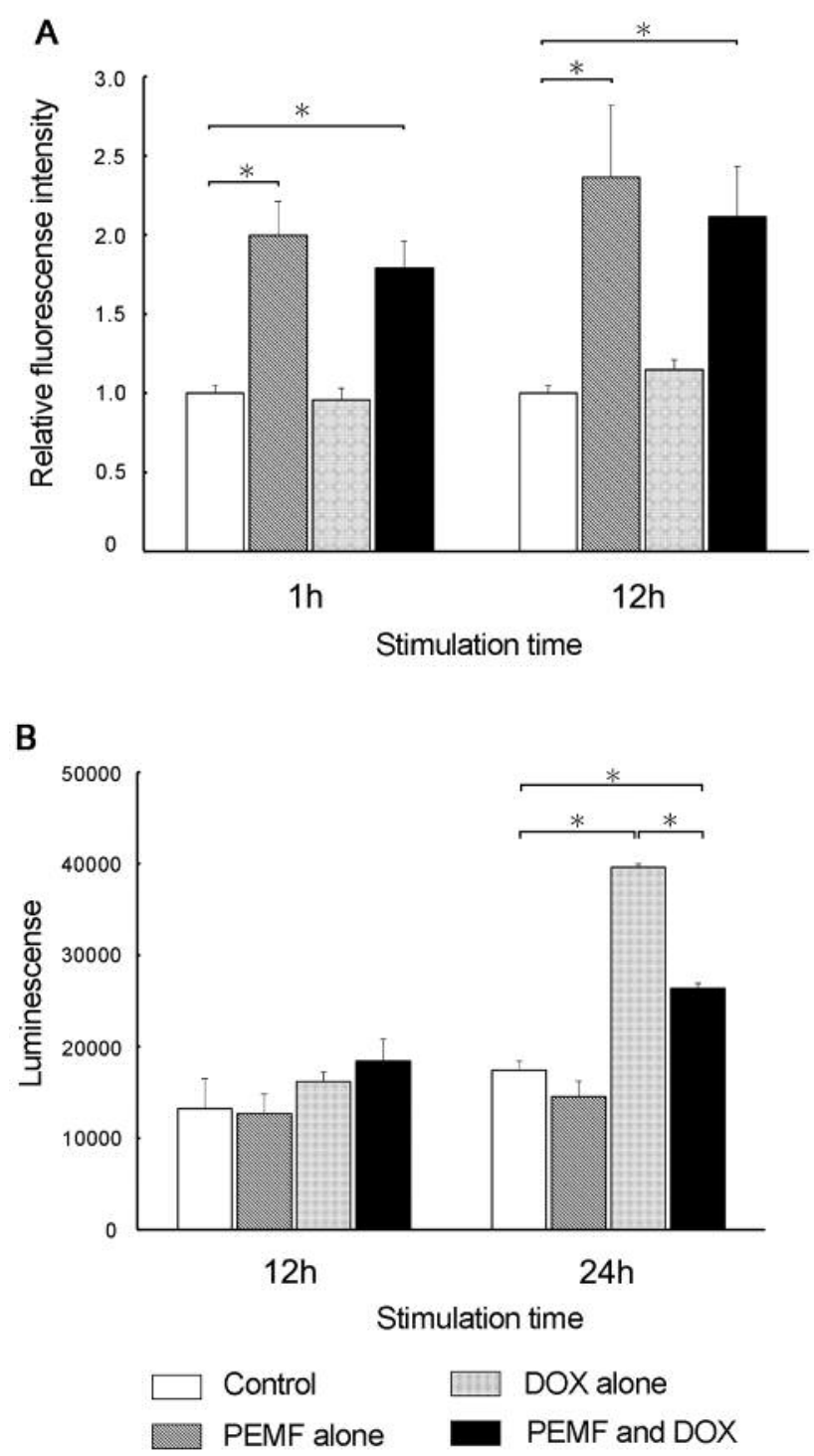

Figure 3. Intracellular apoptosis molecules. A: The kinetics of intracellular $\left[\mathrm{Ca}^{2+}\right] \mathrm{i}$ level after treatment of LM8 cells with a combination of pulsed electromagnetic field (PEMF) and doxorubicin $(D O X)$. The fluorescence intensities were evaluated treatment of LM8 cells with PEMF and DOX, individually and in combination. B: Caspase 3/7 activity in LM8 cells after treatment of PEMF and DOX, $P E M F$ alone, and DOX alone evaluated via luminescence intensity. The data represent means $\pm S . D$. of five independent experiments. ${ }^{*} p<0.05$ $v s$. doxorubicin alone or untreated control.

\section{Discussion}

Doxorubicin has been used as therapy for osteosarcoma since the 1970s (13-15) and subsequently was included in T10 (16, 17) and T12 protocols (18), which are combinations of various anticancer drugs. In Japan, neoadjuvant chemotherapy for osteosarcoma using the above protocols has led to dramatic improvements in the survival rate (19). However, recurrences, metastasis, and drug resistance often occur. It is hoped that a novel therapeutic approach can be developed to improve such poor prognosis. A clinical trial has reported that caffeinepotentiated chemotherapy prolongs the survival of patients with osteosarcoma with pulmonary metastasis (20). Another study revealed that valproic acid, which is a histone deacetylase inhibitor, enhances doxorubicin-induced cytotoxicity in canine osteosarcomas $(21,22)$. We previously reported that PEMF enhances doxorubicin-uptake and induces cytotoxic effects on bovine aortic endothelial cells (12). We believe that a combination of low-intensity PEMF instead of pharmaceutical preparations, such as caffeine and anticancer drugs, might allow use of lower doses of general anticancer drugs. This study aimed to investigate new developments in therapies for osteosarcoma and investigated the synergistic effects of PEMF and doxorubicin.

The $\mathrm{IC}_{50}$ for combined PEMF and doxorubicin was about 2 -fold lower than that of doxorubicin alone, suggesting that PEMF enhanced the antiproliferative activity of doxorubicin. Thus, in support of our previous report (9), which showed PEMF reduced the cell proliferation of another mouse osteosarcoma cell line (Dunn), this study showed that PEMF and doxorubisin appear to have synergistic antiproliferative effect, at least in mouse osteosarcoma. Loja et al. reported that PEMF at the same intensity as that in our study slightly increased cell proliferation in colorectal and ductal carcinoma (Colo-320DM and ZR-75-1) cell lines (23). The discrepancies between our findings and the former reports might depend on the type of cells used.

We hypothesized that the synergistic effect revealed was involved in doxorubicin-derived apoptosis. Firstly, we investigated the effects of PEMF and doxorubicin co-treatment on the cell cycle. Unfortunately, PEMF did not alter the population of cells at $\mathrm{G}_{2} / \mathrm{M}$ mediated by doxorubicin, which agrees with the report by Fan et al. (24), and indicates that PEMF does not have a synergistic effect on the cell cycle. Next, we investigated the effect on the mitochondrial membrane potential, which is a general apoptosis index, and found that potential did not change after 1 or $12 \mathrm{~h}$ of stimulation. The combination of PEMF and doxorubicin raises the possibility that another mechanism excluding the mitochondrial pathway is involved.

Some studies have investigated the relationship between PEMF stimulation and intracellular signaling molecules. The kinetics of $\left[\mathrm{Ca}^{2+}\right]$ i levels under treatment with PEMF have been presented in many reports, and many researchers have focused on undifferentiated cells such as mesenchymal stem cells $(5,25,26)$. The findings show that PEMF increases the $\left[\mathrm{Ca}^{2+}\right]$ i level via $\mathrm{L}$-type voltage $\mathrm{Ca}^{2+}$ channels $(27,28)$. Our results were also in agreement with these of previous studies. Immediately after stimulation with PEMF alone, $\left[\mathrm{Ca}^{2+}\right]$ i levels were up-regulated; however, the mechanism behind this 
A

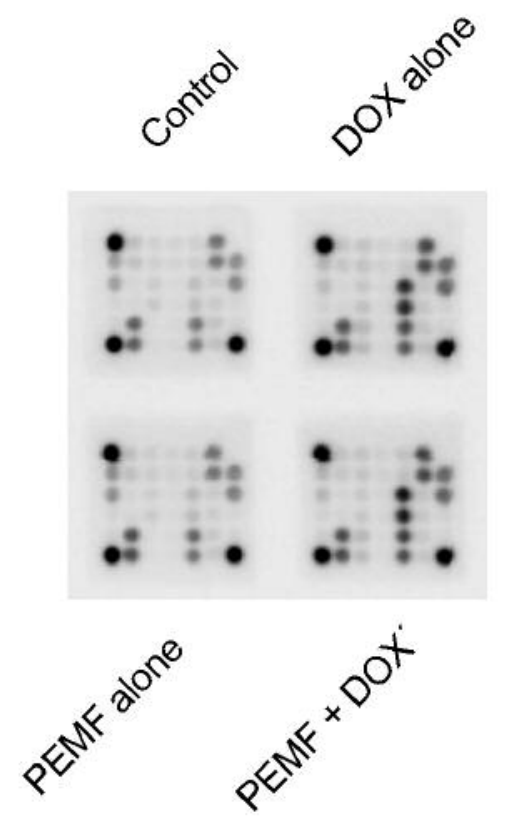

Spot no.

1

2

3

4

5

6

7

8

9

10

10

11

12

13

14

15

16

17

18

19

20

21
Target protein

Positive control

Negative control

Phosphorylated ERK1/2

Phosphorylated AKT

Phosphorylated BAD

Phosphorylated HSP27

Phosphorylated SMAD2

Phosphorylated p53

Phosphorylated p38MAPK

Phosphorylated JNK

PARP

Caspase-3

Caspase-7

Total IkB

Phosphorylated CHK1

Phosphorylated CHK2

Phosphorylated IKB

Phosphorylated elF2a

Phosphorylated TAK1

Survivin

aTubulin

B

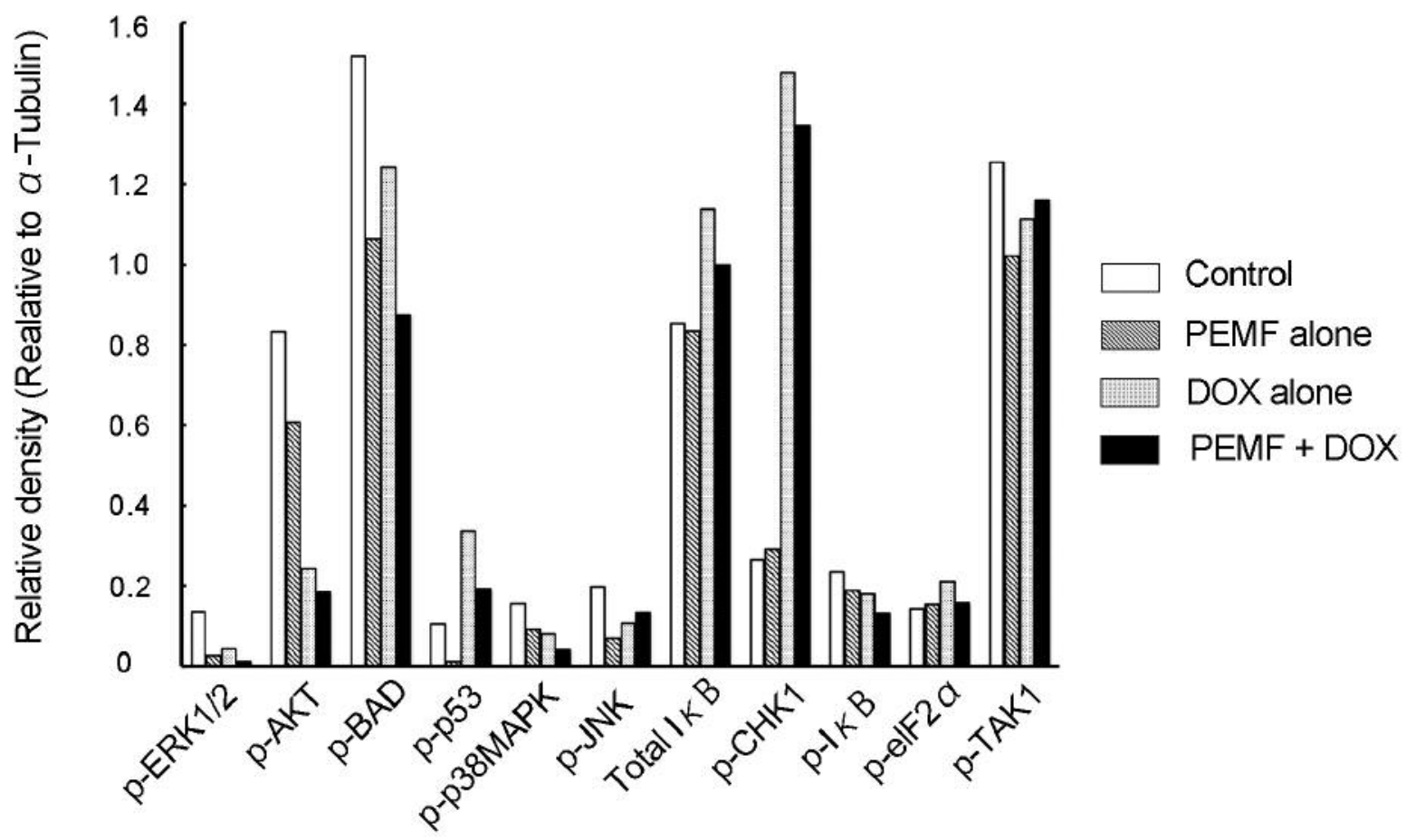

Figure 4. Comprehensive intracellular signaling molecules analysis. A: The stress and apoptosis signaling protein arrays after treatment of LM8 cells with combination of pulsed electromagnetic field (PEMF) and doxorubicin (DOX), PEMF alone, and DOX alone. Of 13 signaling molecules that react to mouse cell lines, six were well recognized. B: The relative densities of the spots of signaling molecules were calculated against $\alpha$ tubulin. The data represent the means of four independent experiments. 
PEMF-regulated $\left[\mathrm{Ca}^{2+}\right] \mathrm{i}$ system remains unknown. Moreover, treatment with doxorubicin alone did not alter the level of $\left[\mathrm{Ca}^{2+}\right] \mathrm{i}$, and the variation in $\left[\mathrm{Ca}^{2+}\right] \mathrm{i}$ level appears to be strongly dependent on PEMF but not on doxorubicin. In any case, this result suggests that the anti-proliferative activity of PEMF and doxorubicin combined is associated with apoptosis and leads to higher $\left[\mathrm{Ca}^{2+}\right] \mathrm{i}$ levels.

Next, we focused on the activity of caspase $3 / 7$, which is an key apoptosis molecule. PEMF for $24 \mathrm{~h}$ moderately inhibited the enhancement of caspase $3 / 7$ activity induced by doxorubicin, although PEMF alone had no effect, which is similar to the results of a neuronal cellular model treated with PEMF (8). Since PEMF and doxorubicin co-treatment for 12 hours showed a slight-increment in caspase 3/7 activity, this combination might have the potential to enhance caspase $3 / 7$ activities between $12 \mathrm{~h}$ and $24 \mathrm{~h}$ after the start of treatment, although these findings do not exclude the possibility of suppression by PEMF. Our findings potentially favor the report by Reihani Kermani et al. which showed that a low frequency of PEMF increases caspase 3 expression in intervertebral discs (29). The antiproliferative effects of PEMF and doxorubicin combined appear to involve caspase $3 / 7$ activity.

In undifferentiated cells, such as bone marrow-derived stromal cells, it is reported that PEMF alone does not affect ERK1/2 phosphorylation (5). Conversely, in cancer cell lines, Sylvester et al. indicated that the expression of both phosphorylated MAPK-kinase-ERK kinase (MEK) that is defined as dual-specificity protein kinases that function in a mitogen activated protein kinase cascade controlling cell growth and differentiation and phosphorylated ERK was enhanced in epithelial CL-S1 cells by PEMF (30). Another report showed that PEMF regulates $B c l 2$ and Aif mRNA in monocytic MonoMac6 cell line $(31,32)$. Thus, the induction of expression of intracellular signaling molecules by PEMF is poorly understood, although the results might depend on the type of cells investigated. At the present time, the intracellular signaling molecules affected by PEMF and doxorubicin co-treatment in osteosarcoma cells remain unclear. We explored ways to identify these molecules and carried out comprehensive analysis using a stress and apoptosis signaling array. PEMF enhanced the reduction of phosphorylated $\mathrm{BAD}$ by doxorubicin, and weakly inhibited the enhanced phosphorylation of CHK1, p53 and total IkB expression mediated by doxorubicin. Our results show that the reduction of phosphorylated BAD expression by PEMF and doxorubicin combined was partially linked to the inhibition of cell growth. In addition, doxorubicin is known to up-regulate p65 NFkB activity (33, 34), but not affect apoptotic target genes. Since p65 NFKB activity is closely linked to $\mathrm{IkB}$, our preliminary experiments investigated the effects on proliferation by doxorubicin and p65 NFkB inhibitor dehydroxymethylepoxyquinomicin
(DHMEQ) in LM8 cells. DHMEQ enhanced the antiproliferative activity of doxorubicin (data not shown). This phenomenon perhaps partially reveals the outcome of a combination of PEMF and doxorubicin, such as the effect on total IkB expression.

In conclusion, a recent report showed that a combination of cisplatin and PEMF inhibited the growth of mouse melanoma B16F10 cells (35), while another study reported that PEMF protected puromycin-induced U937 cell death, depending on cell density (36). Although reports on combined anticancer drug and PEMF therapies are scarce, a combination of PEMF and doxorubicin appears to affect osteosarcoma cells. This combination could serve as a novel therapeutic strategy against osteosarcoma.

\section{Conflicts of Interest}

None.

\section{Acknowledgements}

The Authors thank Professor Kazuo Umezawa of the Department of Molecular Target Medicine Screening for providing the DHMEQ.

\section{References}

1 Fukada E and Yasuda I: On the piezoelectric effect of bone. J Physic Soc Japan 12: 1158-1162, 1957.

2 Shamos MH, Lavine LS and Shamos MI: Piezoelectric effect in bone. Nature 197: 81, 1963.

3 Bassett CA and Becker RO: Generation of electric potentials by bone in response to mechanical stress. Science 137: 1063-1064, 1962.

4 Friedenberg ZB and Brighton CT: Bioelectric potentials in bone. J Bone Joint Surg 48: 915-923, 1966.

5 Jansen JH, van der Jagt OP, Punt BJ, Verhaar JA, van Leeuwen JP, Weinans $\mathrm{H}$ and Jahr $\mathrm{H}$ : Stimulation of osteogenic differentiation in human osteoprogenitor cells by pulsed electromagnetic fields: An in vitro study. BMC Musculoskelet Disord 11: 188, 2010.

6 Caliskan SG, Bilgin MD and Kozaci LD: Effect of pulsed electromagnetic field on MMP-9 and TIMP-1 levels in chondrosarcoma cells stimulated with il-1beta. Asian Pac J Cancer Prev 16: 2701-2705, 2015.

7 Crocetti S, Beyer C, Schade G, Egli M, Frohlich J and FrancoObregon A: Low intensity and frequency pulsed electromagnetic fields selectively impair breast cancer cell viability. PLoS One 8: e72944, 2013.

8 Osera C, Amadio M, Falone S, Fassina L, Magenes G, Amicarelli F, Ricevuti G, Govoni S and Pascale A: Pre-exposure of neuroblastoma cell line to pulsed electromagnetic field prevents $\mathrm{H}_{2} \mathrm{O}_{2}$-induced ros production by increasing mnsod activity. Bioelectromagnetics 36: 219-232, 2015.

9 Miyagi N, Sato K, Rong Y, Yamamura S, Katagiri H, Kobayashi $\mathrm{K}$ and Iwata $\mathrm{H}$ : Effects of pemf on a murine osteosarcoma cell line: Drug-resistant (p-glycoprotein-positive) and non-resistant cells. Bioelectromagnetics 21: 112-121, 2000. 
10 Zhang D, Pan X, Ohno S, Osuga T, Sawada S and Sato K: No effects of pulsed electromagnetic fields on expression of cell adhesion molecules (integrin, CD44) and matrix metalloproteinase-2/9 in osteosarcoma cell lines. Bioelectromagnetics 32: 463-473, 2011.

11 Saito M, Sawada S, Kitamoto K, Akao M and Sato K: Change of an intracelluar calcium concetration in the human osteosarcoma by the PEMF stimulation -in vitro-. J Aichi Med Univ Assoc 41: 1-7, 2013.

12 Ohno S, Zhang D, Osuga T, Sawada S, Saga S, Yoshikawa K and Sato K: Effects of pulsed electromagnetic fields on bovine aortic endothelial cells -chemotherapeutic effcts using PEMF-. J Aichi Med Univ Assoc 35: 7-18, 2007.

13 Gottlieb JA and Fernandez CH: Proceedings: Recent progress in the chemotherapy of primary tumors of bone. Proc Natl Cancer Conf 7: 959-962, 1972.

14 Carter SK: Adriamycin-a review. J Natl Cancer Inst 55: 12651274, 1975.

15 Handelsman $\mathrm{H}$ and Carter SK: Current therapies in osteosarcoma. Cancer Treat Rev 2: 77-83, 1975.

16 Rosen G, Caparros B, Huvos AG, Kosloff C, Nirenberg A, Cacavio A, Marcove RC, Lane JM, Mehta B and Urban C: Preoperative chemotherapy for osteogenic sarcoma: Selection of postoperative adjuvant chemotherapy based on the response of the primary tumor to preoperative chemotherapy. Cancer 49: 1221-1230, 1982.

17 Rosen G, Marcove RC, Caparros B, Nirenberg A, Kosloff C and Huvos AG: Primary osteogenic sarcoma: The rationale for preoperative chemotherapy and delayed surgery. Cancer 43: 2163-2177, 1979.

18 Meyers PA, Gorlick R, Heller G, Casper E, Lane J, Huvos AG and Healey $\mathrm{JH}$ : Intensification of preoperative chemotherapy for osteogenic sarcoma: Results of the memorial sloan-kettering (T12) protocol. J Clin Oncol 16: 2452-2458, 1998

19 Iwamoto Y, Tanaka K, Isu K, Kawai A, Tatezaki S, Ishii T, Kushida K, Beppu Y, Usui M, Tateishi A, Furuse K, Minamizaki $\mathrm{T}$, Kawaguchi $\mathrm{N}$ and Yamawaki S: Multiinstitutional phase II study of neoadjuvant chemotherapy for osteosarcoma (NECO study) in japan: NECO-93J and NECO-95J. J Orthop Sci 14: 397-404, 2009.

20 Kimura H, Tsuchiya H, Shirai T, Nishida H, Hayashi K, Takeuchi A, Ohnari I and Tomita K: Caffeine-potentiated chemotherapy for metastatic osteosarcoma. J Orthop Sci 14: 556-565, 2009.

21 Wittenburg LA, Bisson L, Rose BJ, Korch C and Thamm DH: The histone deacetylase inhibitor valproic acid sensitizes human and canine osteosarcoma to doxorubicin. Cancer Chemother Pharmacol 67: 83-92, 2011.

22 Wittenburg LA, Gustafson DL and Thamm DH: Phase i pharmacokinetic and pharmacodynamic evaluation of combined valproic acid/doxorubicin treatment in dogs with spontaneous cancer. Clin Cancer Res 16: 4832-4842, 2010.

23 Loja T, Stehlikova O, Palko L, Vrba K, Rampl I and Klabusay M: Influence of pulsed electromagnetic and pulsed vector magnetic potential field on the growth of tumor cells. Electromagn Biol Med 33: 190-197, 2014.

24 Fan C, Zheng W, Fu X, Li X, Wong YS and Chen T: Strategy to enhance the therapeutic effect of doxorubicin in human hepatocellular carcinoma by selenocystine, a synergistic agent that regulates the ros-mediated signaling. Oncotarget 5: 28532863,2014
25 Ceccarelli G, Bloise N, Mantelli M, Gastaldi G, Fassina L, De Angelis MG, Ferrari D, Imbriani M and Visai L: A comparative analysis of the in vitro effects of pulsed electromagnetic field treatment on osteogenic differentiation of two different mesenchymal cell lineages. Biores Open Access 2: 283-294, 2013.

26 Schwartz Z, Simon BJ, Duran MA, Barabino G, Chaudhri R and Boyan BD: Pulsed electromagnetic fields enhance BMP-2dependent osteoblastic differentiation of human mesenchymal stem cells. J Orthop Res 26: 1250-1255, 2008.

27 Petecchia L, Sbrana F, Utzeri R, Vercellino M, Usai C, Visai L, Vassalli $\mathrm{M}$ and Gavazzo P: Electro-magnetic field promotes osteogenic differentiation of bm-hmses through a selective action on $\mathrm{Ca}^{2+}$-related mechanisms. Sci Rep 5: 13856, 2015.

28 Li Y, Yan X, Liu J, Li L, Hu X, Sun H and Tian J: Pulsed electromagnetic field enhances brain-derived neurotrophic factor expression through L-type voltage-gated calcium channel- and ERK-dependent signaling pathways in neonatal rat dorsal root ganglion neurons. Neurochem Int 75: 96-104, 2014.

29 Reihani Kermani H, Pourghazi M and Mahani SE: Effects of pulsed electromagnetic field on intervertebral disc cell apoptosis in rats. Electromagn Biol Med 33: 246-249, 2014.

30 Sylvester PW, Shah SJ, Haynie DT and Briski KP: Effects of ultra-wideband electromagnetic pulses on pre-neoplastic mammary epithelial cell proliferation. Cell Prolif 38: 153-163, 2005.

31 Kaszuba-Zwoinska J, Cwiklinska M, Balwierz W, Chorobik P, Nowak B, Wojcik-Piotrowicz K, Ziomber A, Malina-Novak K, Zaraska W and Thor PJ: Changes in cell death of peripheral blood lymphocytes isolated from children with acute lymphoblastic leukemia upon stimulation with $7 \mathrm{~Hz}, 30 \mathrm{mT}$ pulsed electromagnetic field. Cell Mol Biol Lett 20: 130-142, 2015.

32 Kaszuba-Zwoinska J, Chorobik P, Juszczak K, Zaraska W and Thor PJ: Pulsed electromagnetic field affects intrinsic and endoplasmatic reticulum apoptosis induction pathways in MonoMac6 cell line culture. J Physiol Pharmacol 63: 537-545, 2012.

33 Cole MP, Tangpong J, Oberley TD, Chaiswing L, Kiningham KK and St Clair DK: Nuclear interaction between adr-induced p65 and p53 mediates cardiac injury in iNOS (-/-) mice. PLoS One 9: e89251, 2014.

34 Singh RP, Mallikarjuna GU, Sharma G, Dhanalakshmi S, Tyagi AK, Chan DC, Agarwal C and Agarwal R: Oral silibinin inhibits lung tumor growth in athymic nude mice and forms a novel chemocombination with doxorubicin targeting nuclear factor $\mathrm{kB}-$ mediated inducible chemoresistance. Clin Cancer Res 10: 86418647, 2004

35 Kranjc S, Kranjc M, Scancar J, Jelenc J, Sersa G and Miklavcic D: Electrochemotherapy by pulsed electromagnetic field treatment (PEMF) in mouse melanoma B16F10 in vivo. Radiol Oncol 50: 39-48, 2016.

36 Kaszuba-Zwoinska J, Wojcik K, Bereta M, Ziomber A, Pierzchalski P, Rokita E, Marcinkiewicz J, Zaraska W and Thor P: Pulsating electromagnetic field stimulation prevents cell death of puromycin treated u937 cell line. J Physiol Pharmacol 61: 201-205, 2010 